\title{
AREA OF DISCONTINUOUS SURFACES
}

\author{
BY CASPER GOFFMAN ${ }^{1}$
}

Communicated by J. W. T. Youngs, January 19, 1961

1. A general theory of surface area, $[1 ; 2]$, exists for the nonparametric case. Thus, area is defined for all measurable $f$ on the unit square $Q=I \times J$. The area functional is lower semi-continuous with respect to almost everywhere convergence and agrees with the Lebesgue area for continuous $f$. On the other hand, for continuous parametric mappings $T$ of the closed unit square $Q$ into euclidean 3 -space $E_{3}$, Lebesgue area is not lower semi-continuous with respect to almost everywhere convergence nor even, as C. J. Neugebauer has shown, [3], with respect to pointwise convergence.

It thus appears that a theory of parametric surface area must be restricted to surfaces which cannot deviate too far from the ones given by continuous mappings. In this paper, we develop the beginnings of a theory for a class of surfaces which we call linearly continuous.

2. Let $f$ be a real function defined on $Q$ and, for every $u$, let $f_{u}$ be defined by $f_{u}(v)=f(u, v)$ and let $f_{v}$ be defined similarly. Then $f$ is linearly continuous if $f_{u}$ is continuous for almost all $u$ and $f_{v}$ is continuous for almost all v. A mapping $T: x=x(u, v), y=y(u, v)$, $z=z(u, v)$ of $Q$ into $E_{3}$ is linearly continuous if $x, y, z$ are linearly continuous.

A sequence $\left\{f_{n}\right\}$ of functions converges linearly to a function $f$ if $\left(f_{n}\right)_{u}$ converges uniformly to $f_{u}$ for almost all $u$, and $\left(f_{n}\right)_{v}$ converges uniformly to $f_{v}$ for almost all $v$. A sequence $T_{n}: x=x_{n}(u, v), y=y_{n}(u, v)$, $z=z_{n}(u, v)$ converges linearly to a mapping $T: x=x(u, v), y=y(u, v)$, $z=z(u, v)$ if $\left\{x_{n}\right\},\left\{y_{n}\right\},\left\{z_{n}\right\}$ converge linearly to $x, y, z$, respectively.

Let $P$ be the set of quasi linear mappings from $Q$ into $E_{3}$. For $p, q \in Q$ let

$$
\begin{aligned}
& d(p, q)=\inf \left[k: \text { there are sets } A_{k} \subset I, B_{k} \subset J,\right. \\
& \quad m\left(A_{k}\right)>1-k, m\left(B_{k}\right)>1-k, \text { and }|p(u, v)-q(u, v)|<k \\
& \left.\quad \text { on }\left(A_{k} \times J\right) \cup\left(I \times B_{k}\right)\right] .
\end{aligned}
$$

It is easy to verify that $P$ is a metric space and that $\left\{p_{n}\right\}$ converges to $p$ in this space if and only if it converges linearly. Let $E$ be the elementary area functional on $P$. It is not hard to prove

\footnotetext{
${ }^{1}$ Research supported by National Science Foundation Grant No. NSF G-5867.
} 
THEOREM 1. $E$ is lower semi-continuous on $P$. In other words, if $\left\{p_{n}\right\}$ converges linearly to $p$ then $\lim$ inf $E\left(p_{n}\right) \geqq E(p)$.

By the Fréchet extension theorem, $E$ is extended to a lower semicontinuous functional $\Phi$ on the completion $\mathscr{L}$ of $P$.

THEOREM 2. The completion $\&$ of $P$ is the space of linearly continuous mappings with the metric corresponding (as above) to linear convergence.

3. It is obvious that for every continuous mapping $T, A(T) \geqq \Phi(T)$ where $A(T)$ is the Lebesgue area. The inverse inequality holds so that the functional $\Phi$ constitutes a legitimate extension of Lebesgue area to substantially wider class of mappings than the continuous ones. We outline the proof.

For a continuous $T: x=x(u, v), y=y(u, v), z=z(u, v)$, the lower area $V(T)$ is defined as follows:

Let $T_{1}: y=y(u, v), z=z(u, v), T_{2}: x=x(u, v), z=z(u, v)$, and $T_{3}: x=x(u, v), y=y(u, v)$ be the associated flat mappings. For every simple polygonal region $P$ in $Q^{0}$, let

$$
v_{1}(P)=\int\left|O\left(\xi, T_{1} P^{*}\right)\right|
$$

where the integration is over the $y z$ plane, and $O\left(\xi, T_{1} P^{*}\right)$ is the topological index of $T_{1} P^{*}$ at $\xi$ ( $A^{0}$ and $A^{*}$ are the interior and boundary, respectively, of a set $A$ ). Define $v_{2}(P)$ and $v_{3}(P)$, similarly, and let

$$
v(P)=\left[v_{1}(P)^{2}+v_{2}(P)^{2}+v_{3}(P)^{2}\right]^{1 / 2} .
$$

Let $\pi=\left(P_{1}, \cdots, P_{n}\right)$ be a finite set of pair-wise disjoint simple polygonal regions in $Q^{0}$ and

$$
v(\pi)=\sum_{i=1}^{n} v\left(P_{k}\right)
$$

Finally, let

$$
V(T)=\sup [v(\pi): \pi] .
$$
$T$.

Cesari has shown (e.g. [4]) that $A(T)=V(T)$ for every continuous

The distance between 2 sets $A$ and $B$ is defined by

$$
d(A, B)=\sup [d(x, B): x \in A]+\sup [d(y, A): y \in B] .
$$

With this metric, the set $\alpha$ of simple polygonal regions is a separable metric space. Let $\beta \subset \alpha$ be dense in $\alpha$ and 


$$
V_{\beta}=\sup [v(\pi): \pi \subset \beta] .
$$

Lemma 1. $V_{\beta}(T)=V(T)$.

Now, let $\left\{T_{n}\right\}$ be a sequence of continuous mappings which converges linearly to a continuous mapping $T$. Let $\gamma$ be the set of simple polygonal regions whose boundaries consist of line segments parallel to the coordinate axes for which $T$ and $T_{n}, n=1,2, \cdots$ are continuous and on each of which $\left\{T_{n}\right\}$ converges uniformly to $T$. For each $\pi \subset \gamma, \lim \inf v\left(\pi, T_{n}\right) \geqq v(\pi, T)$. Since $\gamma$ is dense in $\alpha$, it follows that $\lim \inf V\left(T_{n}\right) \geqq V(T)$. This proves

THEOREM 3. $A(T)$ is lower semi-continuous with respect to linear convergence on the set of continuous mappings.

Corollary 1. $A(T)=\Phi(T)$ for every continuous $T$.

Proof. For every sequence $\left\{p_{n}\right\}$ of quasi-linear mappings converging linearly to $T, \lim \inf E\left(P_{n}\right) \geqq A(T)$. Choose $\left\{p_{n}\right\}$ so that $\lim E\left(p_{n}\right)=\Phi(T)$. Then $A(T) \leqq \Phi(T)$,

4. A set $S$ will be called negligible if $S \subset Z_{1} \times Z_{2}$ where $Z_{1}$ and $Z_{2}$ have linear measure zero. Kolmogoroff's principle holds in the following form.

TheOREM 4. If $T_{1}$ and $T_{2}$ are linearly continuous mappings from $Q$ into $E_{3}$ and if for every pair of points $\xi, \eta$ not belonging to a negligible set

$$
\left|T_{1} \xi-T_{1} \eta\right| \leqq\left|T_{2} \xi-T_{2} \eta\right|
$$

then $\Phi\left(T_{1}\right) \leqq \Phi\left(T_{2}\right)$.

5. A real function $f$ on $Q$ is BVC if for almost all $u$ and almost all $v, f_{u}$ and $f_{v}$ are equivalent to functions of bounded variation and the corresponding variation functions are summable. $f$ is ACE if for almost all $u$ and almost all $v, f_{u}$ and $f_{v}$ are equivalent to absolutely continuous functions.

For functions which are BVT and ACT it is a simple known fact that the integral means commute with the partial derivatives. This also holds almost everywhere for functions which are BVC and ACE. Using this fact and the fact, [5], that if $f$ is BVC and linearly continuous then the integral means of $f$ converge linearly to $f$, the proof of the following generalization of a theorem of Morrey, [4], may be obtained in somewhat standard fashion. The generalization is in two directions. Instead of holding only for conjugate Lebesgue spaces, the theorem holds for conjugate Köthe spaces, $[6 ; 7]$, and the theorem 
holds for linearly continuous mappings rather than just for continuous ones.

THEOREM 5. If the functions $x, y, z$ of a linearly continuous $T$ are $B V C$ and $A C E$ and if the pairs of partial derivatives $\left(x_{u}, y_{v}\right),\left(x_{v}, y_{u}\right)$, $\left(x_{u}, z_{v}\right),\left(x_{v}, z_{u}\right),\left(y_{u}, z_{v}\right),\left(y_{v}, z_{u}\right)$ belong to conjugate Köthe spaces, the area $\Phi(T)$ is given by the formula

$$
\Phi(T)=\int J d u d v
$$

where $J=\left[J_{1}^{2}+J_{2}^{2}+J_{3}^{2}\right]^{1 / 2}$ and $J_{1}, J_{2}, J_{3}$ are the jacobians of $T_{1}, T_{2}, T_{3}$, respectively.

6. We define an equivalence relation for linearly continuous mappings. $T$ is equivalent to $T^{\prime}\left(T \approx T^{\prime}\right)$ if there are sequences $\left\{p_{n}\right\}$ and $\left\{q_{n}\right\}$ of quasi linear mappings such that, for every $n, p_{n} \approx q_{n}$ in the Lebesgue sense and $\left\{p_{n}\right\}$ converges linearly to $T,\left\{q_{n}\right\}$ converges linearly to $T^{\prime}$.

The following simple facts hold:

(a) The relation " $\approx$ " has the properties of an equivalence relation.

(b) If $T$ and $T^{\prime}$ are continuous and Fréchet equivalent then $T \approx T^{\prime}$.

(c) If $T \approx T^{\prime}$ then $\Phi(T)=\Phi\left(T^{\prime}\right)$.

We refer to an equivalence class as a surface and to its elements as representations.

$D$ mappings, the Dirichlet integral, and almost conformal mappings are defined as for the continuous case, [4], with BVT and ACT replaced by $\mathrm{BVC}$ and $\mathrm{ACE}$.

We say that a mapping $T$ is simple if there is a negligible set $S$ such that $\xi \in Q-S, \eta \in Q-S, \xi \neq \eta$ implies $T(\xi) \neq T(\eta)$.

The following holds:

THEOREM 6. If $T^{\prime}$ is a linearly continuous simple mapping and $\Phi\left(T^{\prime}\right)<\infty$, the surface given by $T^{\prime}$ has a representation $T$, with jacobian $J$, such that

$$
\Phi\left(T^{\prime}\right)=\Phi(T)=\int J d u d v .
$$

CoRollary. Every linearly continuous nonparametric surface of $f$ nite area has a parametric representation $T$, with jacobian $J$, such that

$$
\Phi(T)=\int J d u d v
$$




\section{BIBLIOGRAPHY}

1. L. Cesari, Sulle funzioni a variazione limitata, Ann. Scuola Norm. Sup. Pisa vol. 5 (1936) pp. 299-313.

2. C. Goffman, Lower semi-continuity and area functionals. I, The non-parametric case, Rend. Circ. Mat. Palermo vol. 2 (1954) pp. 203-235.

3. C. J. Neugebauer, Lebesgue area and pointwise convergence, Abstract 554-13, Notices Amer. Math. Soc. vol. 6 (1959) p. 80.

4. L. Cesari, Surface area, Princeton, University Press, 1956.

5. C. Goffman, Area of linearly continuous functions, Acta Math. vol. 103 (1960) pp. $269-290$.

6. J. Dieudonné, Sur les espaces de Köthe, J. Anal. Math. vol. 1 (1951) pp. 81-115.

7. C. Goffman, Completeness in topological vector lattices, Amer. Math. Monthly vol. 66 (1959) pp. 87-92.

Purdue University 